\title{
Building an island in the storm: enhancing the junior doctor experience
}

\author{
Author: Will Owen
}

\begin{abstract}
Aims
To understand the level of satisfaction among junior doctors in our organisation and their priority areas for improvement, and develop a sustainable programme of improvement to enhance their wellbeing and experience.
\end{abstract}

\section{Methods}

We surveyed the junior doctors (both training and non-training grades) in our organisation, asking them to rate their satisfaction with their experience, identify positive and negative aspects of their working lives, and suggest priority areas for improvement. We combined this with information from the General Medical Council national training survey and exception reporting data raised to the guardian of safe working. We identified themes from this data as causes for dissatisfaction and therefore targets for improvement, and then sense-checked these with a focus group of junior doctors from across the organisation.

\section{Results}

We received a low but acceptable $20 \%$ response rate to our survey. Sixty-seven per cent of respondents were either satisfied or very satisfied with their experience. Nineteen per cent were dissatisfied or very dissatisfied and a further $14 \%$ gave a neutral response. Reasons for dissatisfaction grouped in to unsurprising themes: workload and the impact of workload and service demands on training, rotas and access to leave, culture and attitudes towards junior doctors and their training, facilities and environment, and difficulties with induction, contracts and payroll.

With these results we have developed a number of projects to tackle the problem themes identified in the survey and which are delivering sustainable improvements in the junior doctor experience at our organisation. We are focusing on a number of trust-wide areas including; creating appropriate rest facilities for out-of-hours teams in all parts of the hospital and separately making improvements to the doctors' mess, setting up human resources and payroll drop-in sessions to deal with contract and pay issues, improving junior doctor induction processes, developing a training programme for rota coordinators, and developing a 'good practice guide' to help services improve culture and attitudes towards junior doctors and their training.

\section{Conclusion}

Junior doctor morale is at an all-time low. Our vision is to make our organisation an 'island in the storm' of medical training. By engaging with junior doctors and with the support of the executive team in our organisation we have developed a trust-wide programme of work to deliver sustainable improvements to the experience of training and non-training grade junior doctors.

\section{Conflict of interest statement}

None. 